\title{
Combination aquatic and land physical therapy program improved function and reduced spasticity after acute illness and exacerbation of multiple sclerosis: A case report
}

\author{
Jennifer Roos* \\ Department of Physical Therapy, California State University, Fresno, USA
}

\begin{abstract}
Background and purpose: This case report provides retrospective review of an outpatient physical therapy program for a female client with primary progressive multiple sclerosis after acute illness requiring hospitalization that contributed to decline in function and deconditioning. This patient was prescribed a multi-modal intervention that included education and training to restore physical impairments and loss of function with a combination of aquatic and land-based interventions, and introduction to the functional electrical stimulation (FES) cycle.

Case description: 48-year old female with twenty-year history of primary progressive multiple sclerosis seeking conservative management treatment options for spasticity reduction and functional decline post-hospitalization for infection and cellulitis. The client was deconditioned following hospitalization with increased generalized joint pain and weakness, increased spasticity, and increased assistance with daily activities required. At discharge she continued independently with a home program for one-year before re-entering physical therapy for progression of a home program regimen. The client used the FES cycle for a total of twentymonths, including a combination of physical therapy and independent use, as well as multi-modal options including gym and aquatic exercise programs. Extensive training and education was incorporated in all aspects of her therapy program to ensure safety and interval training to ensure patient did not get overheated with exercise.

Outcomes: Subjective reports included improved stamina, increased lower extremity strength, and decreased spasticity with a reduction in pharmacologic management. Bed mobility and sit to stand transfers were modified independent. Emerging stepping strategies were demonstrated with pick-up walker. Able to demonstrate independent set-up of FES cycle and accurately perform gym and aquatic exercise programs by discharge.

Discussion: Compliance with multi-modal interventions demonstrated improved function and allowed client to return to prior level of function. Additional benefits included reduction of pharmacological management for spasticity and emerging gait skills.
\end{abstract}

\section{Background}

Multiple Sclerosis (MS) affects approximately 400,000 people in the United States with approximately 10,000 new cases per year [1]. The national MS society stated that MS is believed to affect approximately 2.3 million people worldwide [2]. MS presents with a wide range of symptoms and onset can usually occur between the ages of 20-50 years old. Symptoms vary and can be intermittent, therefore the disorder can often take years to accurately diagnose in some individuals. Symptoms can include sensory and motor loss, fatigue, heat sensitivity, balance dysfunction, pain, cognitive impairment, depression, spasticity, and sexual dysfunction [3]. Women are diagnosed with MS two and a half times more often than men [4]. "In the United States, a person has a 1:750 risk of developing multiple sclerosis;" MS has a significant impact on the activities of daily living and is influenced by patient's functional capacity, disease progression and symptom management [4].

Spasticity is a common symptom seen in clients with multiple sclerosis. Lance defined spasticity as "a motor disorder characterized by a velocity-dependent increase in tonic stretch reflexes with exaggerated tendon jerks, resulting from hyperexcitability of the stretch reflex" [5]. During physical therapy examination spasticity is commonly measured using the Ashworth scale or the Modified Ashworth scale. The Ashworth scale is commonly used as a test and measure for spasticity. Bohannon and Smith demonstrated significant correlation of the modified Ashworth scale [6].

Deconditioning leads to physical weakness and often results in loss of function. It is common after acute illness and immobility to require increased assistance with daily activities. A client suffering from a chronic condition, such as MS, will see an even greater decline in function and recovery is a slower progression. In general, MS symptoms vary, so an individualized approach to rehabilitation is important. Deconditioning contributes to reduction in overall activity level and can lead to social isolation and decreased self-esteem [7]. Incorporating an exercise program for clients with MS is important to reduce the decline in functional capacity [4]. Healthy people 2020 vision statement defines a society in which all people live long, healthy lives and provide specific objectives stating "physical activity can improve

Correspondence to: Jennifer Roos, Department of Physical Therapy, California State University, Fresno, USA, E-mail: jroos@csufresno.edu

Key words: multiple sclerosis, aquatic therapy, functional electrical stimulation (FES) cycling, spasticity

Received: August 12, 2016; Accepted: August 26, 2016; Published: August 29, 2016 
Roos J (2016) Combination aquatic and land physical therapy program improved function and reduced spasticity after acute illness and exacerbation of multiple sclerosis: A case report

the health and quality of life of Americans of all ages, regardless of the presence of chronic disease or disability [8]." Clients with chronic health conditions may demonstrate a greater decline in function after acute illness due to exacerbation of symptoms related to chronic illness that can increase the time it takes to return to prior functional level.

Functional electrical stimulation (FES) cycling can be used as an ongoing therapy for chronic neurological conditions and as a rehabilitation tool [9]. Multiple studies include clients with a variety of neurologic conditions including; spinal cord injury (SCI), cerebral palsy (CP), stroke, and multiple sclerosis [9-15]. Outcomes of FES cycling include a range of improvements including; relaxation of muscle spasms, prevention or retardation of disuse atrophy, increase local blood circulation, and maintain or increase range of motion [9-15].

The purpose of this case report is to document a multi-modal physical therapy intervention provided to a single client over extended period of time in conjunction with an independent home/gym program. This retrospective case report displays intervention and outcomes using a multi-modal treatment approach including land-based and aquatic interventions. The patient was followed for approximately one and one-half years. Interventions included FES cycling, aquatic therapy, and gym-based exercise program focused on strengthening and cardiovascular components.

\section{Case description}

The client was a 48-year-old Caucasian female diagnosed with primary progressive multiple sclerosis (PPMS) approximately twentyyears ago. She was referred to an outpatient physical therapy clinic for examination following hospitalization due to acute infection secondary to cellulitis caused by a wound. Patient was hospitalized for two days prior to discharge home with a referral from her neurologist for outpatient physical therapy services. Prior functional status included modified independent activities of daily living (ADL) and transfers at wheelchair level. Lived alone in single-story home, able to drive and access her community at a modified independent wheelchair level. Caregiver assistance was required for three hours per day five-six days per week prior to illness and increased following discharge home from hospital. Prior ADL was modified independent with meal preparation and bathroom hygiene, performed from a static standing position in short intervals. Past medical history included three orthopedic injuries and one minor orthopedic surgery over twenty years ago. Medications included; Baclofen, Betaseron, Tizanidine, Diazepam, Nabumetone, Carvedilol, Hydrocodone, and Doxycycline.

\section{Systems review}

Client was alert and oriented X4, demonstrated excellent recall of past medical history and prior level of function without confusion. Cardiopulmonary system was stable with medication management for high blood pressure. Integumentary system was negative, no open wounds or skin infection. Musculoskeletal and neurologic systems were identified as primary systems involved based on history and chart review. During initial exam spasticity was observed in bilateral lower extremities at rest, seated in manual wheelchair, and with movement.

The client's primary goal was to improve stamina, lower extremity strength, improve bilateral shoulder pain, resume intermittent standing ADLs, and initiate ability to take steps with a pickup walker to facilitate ease with transfers and ADLs. Reported complaints of increased spasticity since recent hospitalization and had personal goal to manage spasticity without requiring use of additional medication.

\section{Clinical impression \# 1}

The primary impairment appeared to be generalized weakness, increased spasticity, exacerbation of symptoms related to recent illness and immobility, and her long-standing diagnosis of MS. Client was no longer able to pull herself up to a standing position for daily routine activities and requires additional caregiver assistance in the home as a result of functional decline. Additional information not available at the time of the examination was requested from the primary referring physician, and included recent MRI results.

Plan for initial examination included setting up priority for test and measures considering her fatigue level and spasticity complaints. The client expressed high motivation levels, and prior therapy experiences increased her awareness and expectations.

\section{Examination}

At initial examination patient presented with strength deficits; right lower extremity MMT 0-2+/5, and left lower extremity MMT 2-3/5. Modified Ashworth scale 2+ to 3. Strength testing and the modified Ashworth scale are two commonly used examination methods when reviewing the literature. Manual muscle tests are used consistently by physical therapists during the examination of multiple neurological and orthopedic conditions across different physical therapy settings. The modified Ashworth scale is debated in the literature with multiple studies debating its accuracy. "Currently the modified Ashworth scales remain the primary clinical measures of spasticity [16]." Platz et al. stated the lack of standard guidelines for positioning, performance or scoring can give highly variable results for the Ashworth or modified Ashworth scale [17]. Ansari et al. conclude in their research that the "Ashworth and modified Ashworth scales when used in a standardized procedure and in a clinical situation in which raters were not trained, the reliability of both tests are poor [18].” Although there is conflicting evidence on reliability and validity, the Ashworth and/or modified Ashworth scales are still used regularly by physical therapists.

Assessment in functional mobility included: independence in bed mobility with minimal assist for squat-pivot transfer from wheelchair to bed. Safety and independence in wheelchair management, static and dynamic sitting balance is fair. Pain was confirmed with palpation assessment of bilateral shoulders, and client stated that this chronic problem had improved in the past when performing exercises in the pool regularly. Ambulation was not examined during evaluation since the patient is unable to stand at this time. Increased time intervals between activities were required due to effort exerted with each task and client reporting fatigue.

\section{Clinical impression \#2}

The initial impression was confirmed after completing the evaluation. She presented with lower extremity weakness, lower extremity spasticity that impeded mobility, and an overall decline in stamina. Stamina decline, although not measured by specific test, was identified through client challenges with functional tasks such as transfers. She required additional time and assistance as well as subjective reports of increased fatigue after task completion. The FES cycle was an additional intervention considered for this client because it has been identified in the literature to assist in spasticity control along with potential for building strength and stamin in clients with neurological based disorders. Per Fornusek and Davis, FES cycling demonstrate multiple benefits including: increase muscle mass, increased body mass and tone, increased blood flow, and paralyzed muscles following FES training can revert towards normal becoming 
Roos J (2016) Combination aquatic and land physical therapy program improved function and reduced spasticity after acute illness and exacerbation of multiple sclerosis: A case report

fatigue resistant [19]. This client was highly motivated and interested in looking for new or continued methods of exercise regimens that would help her manage spasticity and get her back to her prior level of function. Based on past tolerance to physical therapy services the plan was to include an aquatic exercise program as well.

\section{Intervention}

The client was seen for ten visits which included home program training, and FES cycling. FES training sessions ranged from twentysixty minutes duration. Distance traveled ranged from two to seven miles with progression of greater distances as therapy sessions progressed. However, client did have shorter riding distances, which occurred, based on a variety of circumstances; availability of equipment, illness, or symptoms of intermittent pain or fatigue. FES stimulation was placed on three muscle groups during each FES cycling session. Quadriceps, hamstrings, and tibialis anterior were targeted in bilateral lower extremities and stimulation intensity was increased to client tolerance. Continuous stimulation was used and a five-minute warm up and cool-down was provided during each session on the FES cycle. Due to greater sensory loss in the right leg, stimulation was given to a level of comfort and in general was slightly higher in the right leg. Pulse width ranged 250 us for tibialis anterior and 350-400 us for quadriceps and hamstring groups bilaterally. Frequency is $47.6 \mathrm{~Hz}$ in all channels. Maximum stimulation for muscle groups range from 22-38 mA (Table 1 and 2).

A study by Yeh et al. states that FES cycling was an effective strategy for reducing muscle tone in patients with stroke [11]. Krause et al., states that some patients with spinal cord injury using FES cycling can have beneficial effects on spastic muscle tone [12]. The client in this case report was trained to independently use the FES cycle during her ten treatment sessions so she could continue with a home program upon discharge. She continued to use the FES cycle for over ten months, at a frequency of two to three times per week for 45-60 minute sessions.

Ten months later, the client re-entered physical therapy for evaluation of her home program and incorporation of an additional aquatic exercise regimen. The client was seen for eleven treatment sessions, which included adaptations to FES bike settings as well.
Long-term goals were to increase intensity of FES settings, initiate aquatic regimen, and improve standing function, or ability to walk with assistive device since spasticity had subjectively improve and client reported improved endurance with ADL's.

Aquatic exercise program was adapted to meet individual needs and included use of adaptive equipment such as Styrofoam dumbbells, paddles, and ankle weights (Table 3). Exercises included strengthening and endurance activities for all extremities, trunk stabilization activities, static and dynamic balance activities, and walking with the use of a rail. She demonstrated a paraparetic gait pattern and required use of rail with both hands and/or PT/PTA in the pool to provide manual assist for balance purposes initially with ambulation. The purpose of equipment choices in the pool was based on upon providing resistance, supporting or assisting patient to ensure safety, and allow progression of exercises to an independent level. The pool temperature range was from 92-94 degrees. Client consented to treatment in the pool and was aware of risk factors associated with overheating. The aquatic therapy program was monitored at all times by PT/PTA to ensure appropriate response to activities without fatigue or overheating. Aquatic exercises were taught to the patient in interval training mode to ensure appropriate rest breaks. A gym program included strengthening exercises (Figure 1). FES cycling updates included parameter modifications to include interval training with 5-minute stimulation, on/off cycles. A variety of exercise options were provided during physical therapy instruction to include multi-modal programs. Subjective reports include preference to FES cycling for greatest benefit of spasticity management [13-15].

\section{Outcomes}

When the client was discharged from the initial ten physical therapy sessions she was able to demonstrate improved functional mobility, able to self-propel wheelchair with decreased bilateral shoulder symptoms, and perform a squat pivot transfer modified independent. Client subjectively reported improved spasticity and increased function of lower extremities with daily tasks at home in standing. Her short and long-term goals were met and patient was ready to continue with her home/gym program and the FES cycle program independently.

Following the second bout of physical therapy intervention, ten

Table 1. Test and measures.

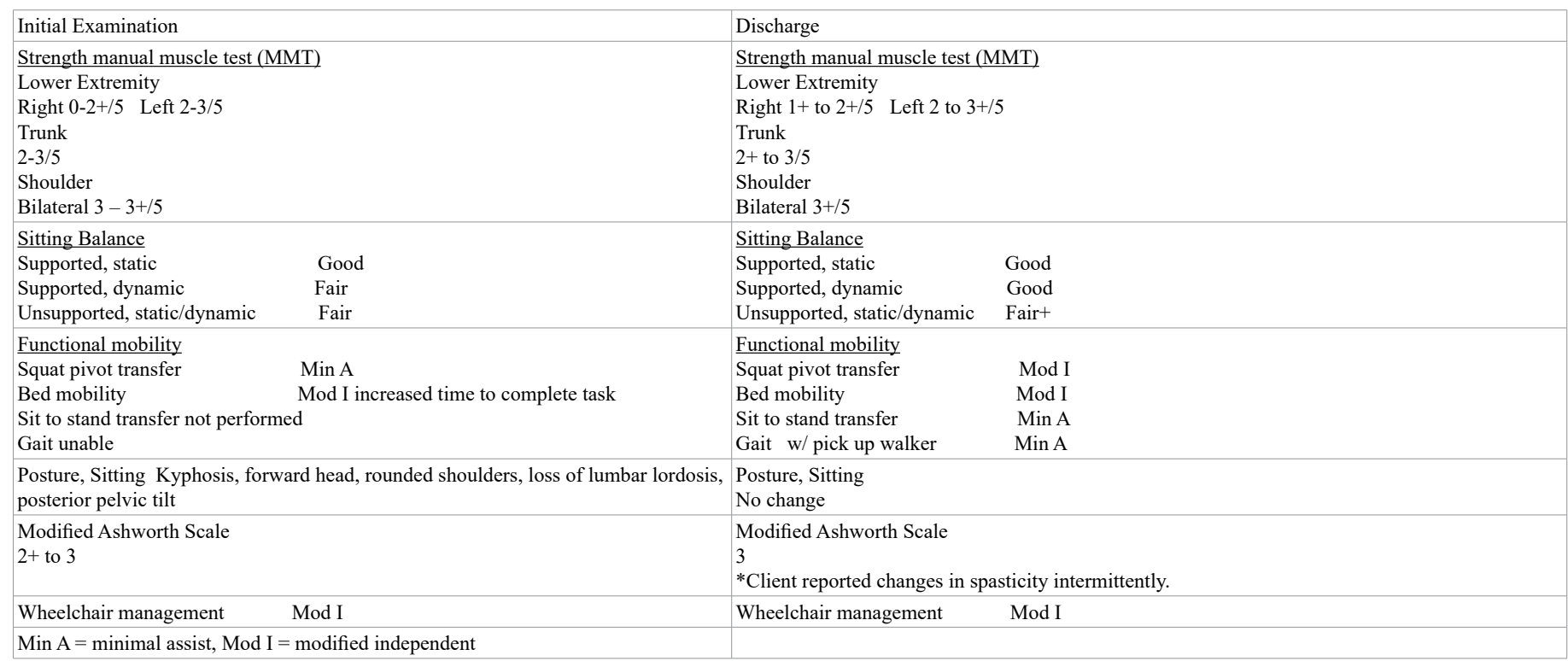


Roos J (2016) Combination aquatic and land physical therapy program improved function and reduced spasticity after acute illness and exacerbation of multiple sclerosis: A case report

Table 2. Fes Cycle Parameters.

\begin{tabular}{|l|l|l|}
\hline Fes Cycle Parameters & Initial parameters & Modified parameters \\
\hline $\begin{array}{l}\text { Electrode Placement } \\
\text { Pulse Width }\end{array}$ & $\begin{array}{l}\text { Quadriceps 350-400 us } \\
\text { Hamstrings 350-400 us } \\
\text { Tibialis Anterior 250 us }\end{array}$ & \\
\hline Warm-Up & 5 MIN (NO STIM) & \\
\hline Cool Down & 5 MIN (NO STIM) & INTERVAL TRAINING \\
\hline Stimulation Mode & CONTINUOUS & 5-min. stim ON/OFF cycle \\
\hline Stimulation Frequency & $47.6 \mathrm{~Hz}$ in all channels & \\
\hline Duration & $20-60$ minute range & \\
\hline Distance & $2-7$ miles per session & \\
\hline Intensity & $\begin{array}{l}\text { Patient tolerance } \\
\text { Max Stimulation range } \\
22-38 \text { mA }\end{array}$ & \\
\hline Sessions & $\begin{array}{l}10 \text { sessions } \\
2 \text { times per week }\end{array}$ & \\
\hline
\end{tabular}

Table 3. Aquatic Exercise Regimen (exercise reps were given to client tolerance and sets determined based on patient stamina and subjective feedback).

Pool entry/exit - chair lift

Transfer Min A, set up of equipment

LE Strengthening

Standing heel raise

Standing unilateral hip flexion

Standing unilateral hip abduction

Trunk stabilization activities

Static standing

Static standing pelvic tilt

Locomotion

Forward walk

Backward walk

Side-step

Seated Stabilization activities

B UE shoulder horizontal Abduction/Adduction

B UE shoulder flexion to 90 degrees

B symmetrical UE row

B alternating UE row

B symmetrical UE tricep press

$B$ alternating UE tricep press

Stretch

Manual gastrocnemius stretch in sitting position

Dynamic Trunk Activities

LE leg press with UE support/rail

LE bicycle with UE support/rail

LE bicycle with noodle in "saddle" position

Abbreviations: $\mathrm{B}=$ bilateral, $\mathrm{UE}=$ upper extremity, $\mathrm{LE}=$ lower extremity

$* 2 \mathrm{lb}$. ankle weights applied to each ankle to aid in reducing buoyancy effects

Table 4. Gym/Home program progression.

\begin{tabular}{|c|c|c|c|}
\hline Exercise & Visit 1 & Visit 2 & Visit 3 \\
\hline Chest Press 10\# & 0 & $3 X 10$ reps. & $3 \times 10$ reps. \\
\hline Lat. Pulls 25\# & $3 \times 10$ reps. & $3 \times 10$ reps. & $3 \times 10$ reps. \\
\hline Tricep Press $15 \#$ & $3 \times 10$ reps. & $3 \times 10$ reps. & $3 \times 10$ reps. \\
\hline Bicep Curls 10\# & $3 \times 10$ reps. & $3 \times 10$ reps & $3 X 10$ reps. \\
\hline UBE & 3 minutes & 5 minutes & 5 minutes \\
\hline Seated Hip adduction $10 \#$ & $2 \times 10$ reps. & $\begin{array}{l}\text { 1X5 reps. } \\
1 \mathrm{X} 10 \text { reps. }\end{array}$ & $2 \mathrm{X} 10$ reps. \\
\hline Seated Hip abduction $10 \#$ & $3 X 5$ reps & 0 & 11 reps. \\
\hline Seated Leg extension 10\# & $2 \times 10$ reps. & 0 & $3 X 10$ reps. \\
\hline Standing Squats & 5 reps & 0 & $2 X 5$ reps. \\
\hline
\end{tabular}

Reps. $=$ Repetitions, Lat. $=$ Latissimus, $\mathrm{UBE}=$ upper body ergometer

months later, the client reported decreased fatigue with ADL's, and decreased use of spasticity medication. Lower extremity strength MMT; right $1+$ to $3+/ 5$, and left 2 to $3+/ 5$. Modified Ashworth scale remained 3 ; the client subjectively reported that her spasticity varied and the day of examination she was experiencing increased symptoms.
Multiple studies provide evidence-based literature about conflicting outcomes with this measurement tool, however it remains one of the most commonly used assessment tools for spasticity [16-18]. Sit to stand transfer required minimal assistance and required pick up walker. Client ambulated ten feet with a pick up walker, minimal assist 


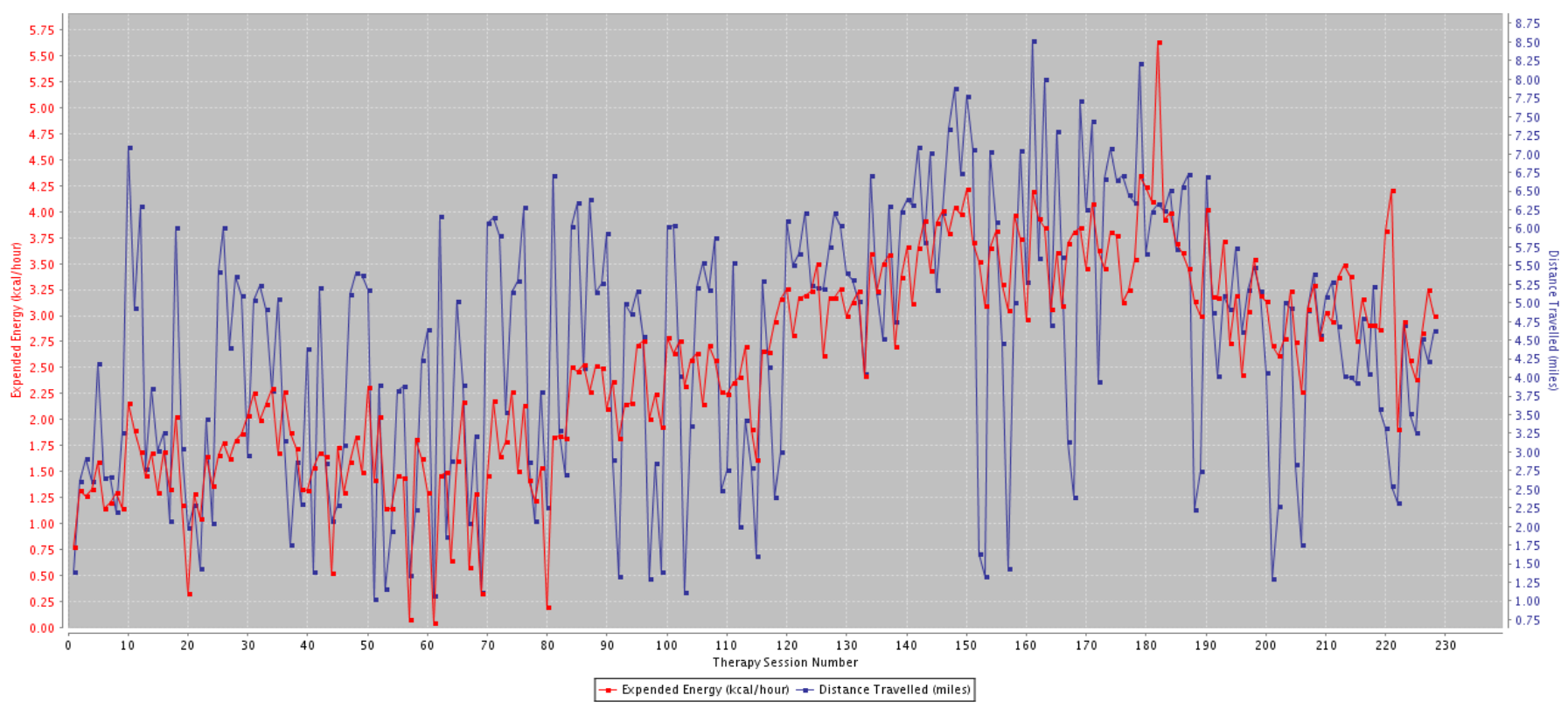

This chart shows distance travelled in BLUE (miles) and expended energy in RED (kcal/hour).

220+ FES cycling sessions over a 20 month-interval. There is continued progression of distance and energy expenditure until patient reaches therapy session \#190. A decrease in distance travelled is observed, with increased expended energy. The change occurred with updates to cycling regimen.

\section{Figure 1. Therapy Progress Graph-RT300.}

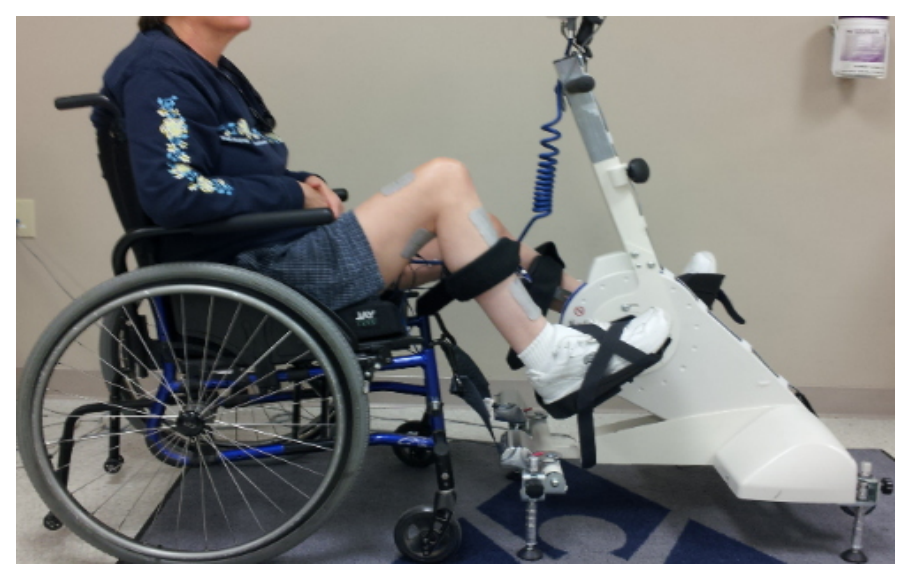

Image

of physical therapist, demonstrating a paraparetic gait pattern. This was the first attempt to take steps in over seven years.

Diagnostic imaging was made available by the client and included MRI findings that reported no new lesions, no active plaque identified, and a few of the foci slightly improved and demonstrate slightly decreased T2 signal intensity when compared to brain MRI one year ago. Spine images state findings are overall stable and without evidence of discrete lesions (Image 1).

Overall, reported benefit from use of FES cycle for multiple purposes included: improved stamina, increased lower extremity strength, spasticity control, and preferred form of exercise for overall health management.

\section{Discussion}

The aquatic exercise regimen provided this client the ability to mobilize in an environment that allowed for higher-level activities and strengthening as well as provide some relief to bilateral shoulder pain. Ambulation activities in the pool were challenging but effort decreased over time. Initially she required assistance by PT/PTA in the pool with ambulation and exercises due to inability to maintain balance and upright posturing during upper or lower extremity activities. Gradual progression from unilateral to bilateral upper and lower extremity exercises increased activation of the core musculature. Client demonstrated improved balance and ability to perform exercises with reduced cueing over time and increased speed. Subjective response included reduced complaints of spasticity after completing physical therapy visits (with FES cycle, land, and aquatic exercises).

Donaldson et al. published a single-subject pilot study for a patient with an incomplete T11-12 spinal cord injury who received FES neuromuscular education from FES cycle ten years after injury, with remarkable recovery [13].

Alon et al. is the first to demonstrate a study with such a high demand of FES cycling. His study included 30 minutes of FES combined with volitional cycling against resistance for 24 sessions, with patient's attempting to keep pace at $60 \mathrm{rpm}$ [14]. Patients did not demonstrate signs of stress on the cardiovascular, pulmonary, or neuromuscular systems. This study focused on motorized cycling in a seated position, a training environment not specific for ambulation, but the gains in walking velocity were similar to data from studies using FES during ambulation [14].

A pilot study done by Krause, et al., reviewed the case of a 46 -year old man who has been diagnosed with MS over 20 years ago. In his study the patient had reduced spastic muscle tone immediately following FES cycle workout. Krause et al. reported reduced tone lasting for "several hours" following treatment session, however a limitation to this study is that there were not a lot of treatment sessions recorded [15].

\section{Conclusion}

This client had been independently using the FES cycle for over 20 
Roos J (2016) Combination aquatic and land physical therapy program improved function and reduced spasticity after acute illness and exacerbation of multiple sclerosis: A case report

months, and at the time she entered physical therapy services, there were no studies published for patient/client(s) with MS, utilizing FES cycles for this extended length of time with a combination of aquatic and land therapy. Client is able to manage spasticity, bear weight in standing for brief intervals during ADL's, and demonstrated gait with assistive device up to ten feet for the first time in over seven years after initiating a multi-modal physical therapy program [19].

Literature states that clients with MS will benefit from regular exercise. Dalgas et al., state that there are only two studies that show promising results for comparing combined resistance and endurance training effects in patients with MS. However, Dalgas et al. also stated that studies comparing longer training intervals ( $>12$ weeks) would be beneficial [20]. The outcome seen in this case report would infer that there is benefit with long-term exercise, however further research for long-term resistance training is needed. This case report demonstrated that this client was using exercise in conjunction with pharmacological management to control her symptoms. By exercising regularly and consistently, this client was able to reduce the amount of medication needed to control spasticity. Future studies of long-term exercise programs, available to patients with chronic neurological conditions, in the community would be important. Also, studies comparing the benefits of long-term exercise programs vs. pharmacologic agents used for symptom management of clients with chronic diseases would be important for future research. A systematic review by Rae-Grant et al identified that there is need for more rigorous studies on "selfmanagement strategies" for chronic neurological conditions, especially MS [21].

\section{Acknowledgement}

This paper was written with proper consent obtained by the client and San Joaquin Valley Rehabilitation Hospital, Fresno CA.

\section{References}

1. https://multiplesclerosis.net/what-is-ms/statistics accessed 1/25/2016.

2. http://www.nationalmssociety.org/What-is-MS/Who-Gets-MS accessed 1/25/2016.

3. Rae-Grant AD, Turner AP, Sloan A, Miller D, Hunziker J, et al. (2011) Self-management in neurological disorders; Systematic review of the literature and potential interventions in multiple sclerosis. J Rehabil Res Dev 29: 197-219.

4. White LJ, Dressendorfer RH (2004) Exercise and multiple sclerosis. Sports Med 34: 1077-1100. [Crossref]

5. Lance JW (1998) Symposium synopsis. In: Feldman RG, Young RR, Koella WP(Eds.), Spasticity: disordered motor control. Chicago: Yearbook Medical., 485-494.
6. Bohannon RW, Smith MB (1987) Interrater reliability of a modified Ashworth scale of muscle spasticity. Phys Ther 67: 206-207. [Crossref]

7. Johnston TE, Wainwright SF (2011) Cycling with functional electrical stimulation in an adult with spastic diplegic cerebral palsy. Phys Ther 91: 970-982. [Crossref]

8. https://healthypeople.gov accessed 6/3/2016.

9. Sosnoff J, Motl RW, Snook EM, Wynn D (2009) Effect of a 4-week period of unloaded leg cycling exercise on spasticity in multiple sclerosis. NeuroRehabilitation 24: 327 331. [Crossref]

10. Ratchford JN, Shore W, Hammond ER, Rose JG, Rifkin R, et al. (2010) A pilot study of functional electrical stimulation cycling in progressive multiple sclerosis. NeuroRehabilitation 27: 121-128. [Crossref]

11. Yeh CY, Tsai KH, Su FC, Lo HC (2010) Effect of a bout of leg cycling with electrical stimulation on reduction of hypertonia in patients with stroke. Arch Phys Med Rehabil 91: 1731-1736. [Crossref]

12. Krause P, Szecsi J, Straube A (2008) Changes in spastic muscle tone increase in patients with spinal cord injury using functional electrical stimulation and passive leg movements. Clin Rehabil 22: 627-634. [Crossref]

13. Donaldson N, Perkins TA, Fitzwater R, Wood DE, Middleton F (2000) FES cycling may promote recovery of leg function after incomplete spinal cord injury. Spinal Cord 38: 680-682. [Crossref]

14. Alon G, Conroy V, Donner T (2011) Intensive training of subjects with chronic hemiparesis on a motorized cycle combined with functional electrical stimulation (FES): a feasibility and safety study. Physiotherapy Res Int 16: 81-91. [Crossref]

15. Krause P, Szecsi J, Straube A (2007) FES cycling reduces spastic muscle tone in patient with multiple sclerosis. Neuro Rehabilitation 22: 335-337. [Crossref]

16. Pandyan AD, Johnson GR, Price CI, Curless RH, Barnes MP, et al. (1999) A review of the properties and limitations of the Ashworth and modified Ashworth Scales as measures of spasticity. Clin Rehabil 13: 373-383. [Crossref]

17. Platz T, Eickhof C, Nuyens G, Vuadens P (2005) Clinical scales for the assessment of spasticity, associated phenomena, and function: a systematic review of the literature. Disabil Rehabil 27: 7-18. [Crossref]

18. Ansari NN, Naghdi S, Moammeri H, Jalaie S (2006) Ashworth Scales are unreliable for the assessment of muscle spasticity. Physiother Theory Pract 22: 119-125. [Crossref]

19. Fornusek C, Davis GM (2004) Maximizing muscle force via low-cadence functional electrical stimulation cycling. J Rehabil Med 36: 232-237. [Crossref]

20. Dalgas U, Stenager E, Ingemann-Hansen T (2008) Review: Multiple sclerosis and physical exercise: recommendations for the application of resistance-, endurance- and combined training. Mult Scler 14: 35-53. [Crossref]

21. Rae-Grant A, Turner A, Sloan A, Miller D, Hunziker J, et al. (2011) Self-management in neurological disorders: Systematic review of the literature and potential interventions in multiple sclerosis care. J Rehabil Res Dev 48: 1087-1100. [Crossref]

Copyright: (C2016 Roos J. This is an open-access article distributed under the terms of the Creative Commons Attribution License, which permits unrestricted use, distribution, and reproduction in any medium, provided the original author and source are credited. 